\title{
Weighted Composition Operators from Hardy to Zygmund Type Spaces
}

\author{
Shanli Ye and Zhengyuan Zhuo \\ Department of Mathematics, Fujian Normal University, Fuzhou 350007, China \\ Correspondence should be addressed to Shanli Ye; ye_shanli@yahoo.com.cn
}

Received 20 January 2013; Accepted 24 March 2013

Academic Editor: Yansheng Liu

Copyright (c) 2013 S. Ye and Z. Zhuo. This is an open access article distributed under the Creative Commons Attribution License, which permits unrestricted use, distribution, and reproduction in any medium, provided the original work is properly cited.

\begin{abstract}
This paper aims at studying the boundedness and compactness of weighted composition operator between spaces of analytic functions. We characterize boundedness and compactness of the weighted composition operator $u C_{\phi}$ from the Hardy spaces $H^{p}$ to the Zygmund type spaces $\mathscr{Z}_{\alpha}=\left\{f \in H(D): \sup _{z \in D}\left(1-|z|^{2}\right)^{\alpha}\left|f^{\prime \prime}(z)\right|<\infty\right\}$ and the little Zygmund type spaces $\mathscr{Z}_{\alpha, 0}$ in terms of function theoretic properties of the symbols $u$ and $\phi$.
\end{abstract}

\section{Introduction}

Let $D=\{z:|z|<1\}$ be the open unit disk in the complex plane $\mathbf{C}$ and $T=\{z:|z|=1\}$ its boundary, and $H(D)$ denotes the set of all analytic functions on $D$. An analytic self-map $\varphi: D \rightarrow D$ induces the composition operator $C_{\varphi}$ on $H(D)$, defined by $C_{\varphi}(f)=f(\varphi(z))$ for $f$ analytic on $D$. It is a wellknown consequence of Littlewood's subordination principle that the composition operator $C_{\varphi}$ is bounded on the classical Hardy $H^{p}(0<p \leq \infty)$ spaces, Bergman $A^{p}(0<p \leq \infty)$ spaces, and Bloch spaces (see, e.g., [1-4]).

Let $u$ be a fixed analytic function on the open unit disk. Define a linear operator $u C_{\varphi}$ on the space of analytic functions on $D$, called a weighted composition operator, by $u C_{\varphi} f=u \cdot(f \circ \varphi)$, where $f$ is an analytic function on $D$. We can regard this operator as a generalization of a multiplication operator and a composition operator. In recent years the weighted composition operator has received much attention and appears in various settings in the literature. For example, it is known that isometries of many analytic function spaces are weighted composition operators (see [5], for instance). Their boundedness and compactness have been studied on various Banach spaces of analytic functions, such as Hardy, Bergman, BMOA, Bloch-type, and Zygmund spaces; see, for example, [6-11]. Also, it has been studied from one Banach space of analytic functions to another; one may see [12-23].
The purpose of this paper is to consider the weighted composition operators from the Hardy space $H^{p}(0<p<$ $\infty)$ to the Zygmund type spaces $\mathscr{Z}_{\alpha}$. Our main goal is to characterize boundedness and compactness of the operators $u C_{\varphi}$ from $H^{p}$ to $\mathscr{Z}_{\alpha}$ in terms of function theoretic properties of the symbols $u$ and $\varphi$.

Now we give a detailed definition of these spaces. For $0 \leq$ $r<1, f(z) \in H(D)$, we set

$$
\begin{gathered}
M_{p}(r, f)=\left(\frac{1}{2 \pi} \int_{0}^{2 \pi}\left|f\left(r e^{i \theta}\right)\right|^{p} d \theta\right)^{1 / p}, \quad 0<p<\infty, \\
M_{\infty}(r, f)=\max _{0 \leq \theta \leq 2 \pi}\left|f\left(r e^{i \theta}\right)\right| .
\end{gathered}
$$

For $0<p \leq \infty$, the Hardy space $H^{p}$ consists of those functions $f \in H(D)$, for which

$$
\|f\|_{p}=\sup _{0 \leq r<1} M_{p}(r, f)<\infty .
$$

It is well known that with norm (2) the $H^{p}$ space is a Banach space if $1 \leq p \leq \infty$, for $0<p<1, H^{p}$ space is a nonlocally convex topological vector space, and $d(f, g)=\|f-g\|_{p}^{p}$ is a complete metric for it. For more information about the $H^{p}$ space, one may see these books, for example, [24, 25]. 
For $\alpha>0$ the $\alpha$-Bloch space $\beta_{\alpha}$ consists of all analytic functions $f$ defined on $D$ such that

$$
\|f\|_{\beta_{\alpha}}=\sup \left\{\left(1-|z|^{2}\right)^{\alpha}\left|f^{\prime}(z)\right|: z \in D\right\}, \quad 0<\alpha<+\infty .
$$

The space $\mathscr{Z}_{\alpha}$ consists of all analytic functions $f$ defined on $D$ such that

$$
\|f\|_{\mathscr{Z}_{\alpha}}=\sup \left\{\left(1-|z|^{2}\right)^{\alpha}\left|f^{\prime \prime}(z)\right|: z \in D\right\}, \quad 0<\alpha<+\infty .
$$

When $\alpha=1$, it is called the Zygmund space. From a theorem by Zygmund (see [26, vol. I, p. 263] or [24, Theorem 5.3]), we see that $f \in \mathscr{Z}_{1}$ if and only if $f$ is continuous in the close unit $\operatorname{disk} \bar{D}=\{z:|z| \leq 1\}$ and the boundary function $f\left(e^{i \theta}\right)$ such that

$$
\frac{\left|f\left(e^{i(\theta+h)}\right)+f\left(e^{i(\theta-h)}\right)-2 f\left(e^{i \theta}\right)\right|}{h}<\infty .
$$

When $\alpha>1$, from Proposition 8 of [27], we know that $\mathscr{Z}_{\alpha}=$ $\beta_{\alpha-1}$. Then the space $\mathscr{Z}_{\alpha}$ is called a Zygmund type space if $0<\alpha \leq 1$. However, all results in this paper are valid for all $\mathscr{Z}_{\alpha}$ spaces $(\alpha>0)$. An analytic function $f \in H(D)$ is said to belong to the little Zymund type space $\mathscr{Z}_{\alpha, 0}$ which consists of all $f \in \mathscr{Z}_{\alpha}$ satisfying $\lim _{|z| \rightarrow 1^{-}}\left(1-|z|^{2}\right)^{\alpha}\left|f^{\prime \prime}(z)\right|=0$. It can be easily proved that $\mathscr{Z}_{\alpha}$ is a Banach space under the norm

$$
\|f\|_{*}=|f(0)|+\left|f^{\prime}(0)\right|+\|f\|_{\mathscr{Z}_{\alpha}} .
$$

And the polynomials are norm-dense in closed subspace $\mathscr{Z}_{\alpha, 0}$. For some other information on this space and some operators on it, see, for example, [28-31].

Throughout this paper, constants are denoted by $C, C(p)$, they are positive, and $C(p)$ are only depending on $p$ and may differ from one occurrence to the another.

\section{Auxiliary Results}

In order to prove the main results of this paper. We need some auxiliary results. The first lemma is well known.

Lemma 1 (see $[24$, p. 65]). For $p>1$, there exists a constant $C(p)$ such that

$$
\int_{0}^{2 \pi} \frac{d \theta}{|1-z|^{p}} \leq \frac{C(p)}{\left(1-|z|^{2}\right)^{p-1}} \quad \text { for every } z \in D
$$

Lemma 2. Suppose that $0<p<\infty, f \in H^{p}$; then

$$
\left|f^{(n)}(z)\right| \leq \frac{\|f\|_{p}}{\left(1-|z|^{2}\right)^{1 / p+n}}
$$

for every $z \in D$ and all nonnegative integer $n=0,1,2, \ldots$

Proof. We use induction on $n$. The case $n=0$ holds because it is Exercise 5 in $[25$, p. 85]. Assume the case $n=k$ holds. Fix $0<r<1$ and let $g(z)=f^{(k)}(r z)$. Then $g(z)$ is in $H^{\infty} \subset \beta_{1}$, and $\|g\|_{\beta_{1}} \leq\|g\|_{\infty}$. It follows that

$$
\begin{aligned}
\left(1-|z|^{2}\right)\left|g^{\prime}(z)\right| & =\left(1-|z|^{2}\right)\left|r f^{(k+1)}(r z)\right| \\
& \leq \frac{\|f\|_{p}}{\left(1-|r z|^{2}\right)^{1 / p+k}} \\
& \leq \frac{\|f\|_{p}}{\left(1-|z|^{2}\right)^{1 / p+k}} .
\end{aligned}
$$

Let $r \rightarrow 1^{-}$; we have

$$
\left|f^{(k+1)}(z)\right| \leq \frac{\|f\|_{p}}{\left(1-|z|^{2}\right)^{1 / p+k+1}}
$$

Then the case $n=k+1$ holds. Hence (8) holds.

Lemma 3. For $0<p<\infty$, suppose $u C_{\varphi}: H^{p} \rightarrow \mathscr{Z}_{\alpha, 0}$ is a bounded operator. Then $u C_{\varphi}: H^{p} \rightarrow \mathscr{Z}_{\alpha}$ is a bounded operator.

This is obvious.

\section{Boundedness of $u C_{\varphi}$ from $H^{p}(0<p<\infty)$ to $\mathscr{Z}_{\alpha}$ and $\mathscr{Z}_{\alpha, 0}$}

In this section we characterize bounded weighted composition operators from the Hardy space $H^{p}(0<p<\infty)$ to the Zygmund spaces $\mathscr{Z}_{\alpha}$.

Theorem 4. Let $\alpha>0,0<p<\infty$, and $u$ be an analytic function on the unit disc $D$ and $\varphi$ an analytic self-map of $D$. Then $u C_{\varphi}$ is a bounded operator from $H^{p}$ to the Zygmund spaces $\mathscr{Z}_{\alpha}$ if and only if the following are satisfied:

$$
\begin{gathered}
\sup _{z \in D} \frac{\left(1-|z|^{2}\right)^{\alpha}\left|u^{\prime \prime}(z)\right|}{\left(1-|\varphi(z)|^{2}\right)^{1 / p}}<\infty, \\
\sup _{z \in D} \frac{\left(1-|z|^{2}\right)^{\alpha}\left|u(z)\left(\varphi^{\prime}(z)\right)^{2}\right|}{\left(1-|\varphi(z)|^{2}\right)^{1 / p+2}}<\infty, \\
\sup _{z \in D} \frac{\left(1-|z|^{2}\right)^{\alpha}\left|2 \varphi^{\prime}(z) u^{\prime}(z)+\varphi^{\prime \prime}(z) u(z)\right|}{\left(1-|\varphi(z)|^{2}\right)^{1 / p+1}}<\infty .
\end{gathered}
$$

Proof. Suppose $u C_{\varphi}$ is bounded from $H^{p}$ to the Zygmund spaces $\mathscr{Z}_{\alpha}$. Then we can easily obtain the following results by taking $f(z)=1$ and $f(z)=z$ in $H^{p}$, respectively:

$$
\begin{aligned}
& u \in \mathscr{Z}_{\alpha}, \\
& \sup _{z \in D}\left(1-|z|^{2}\right)^{\alpha}\left|2 \varphi^{\prime}(z) u^{\prime}(z)+\varphi^{\prime \prime}(z) u(z)+\varphi(z) u^{\prime \prime}(z)\right| \\
& <+\infty .
\end{aligned}
$$


By (14) and the boundedness of the function $\varphi(z)$, we get

$$
K_{1}=\sup _{z \in D}\left(1-|z|^{2}\right)^{\alpha}\left|2 \varphi^{\prime}(z) u^{\prime}(z)+\varphi^{\prime \prime}(z) u(z)\right|<+\infty .
$$

Let $f(z)=z^{2}$ in $H^{p}$ again; in the same way we have

$$
\begin{aligned}
\sup _{z \in D}\left(1-|z|^{2}\right)^{\alpha} \mid & 4 \varphi(z) \varphi^{\prime}(z) u^{\prime}(z)+\varphi^{2}(z) u^{\prime \prime}(z) \\
& +2 u(z)\left(\varphi(z) \varphi^{\prime \prime}(z)+\left(\varphi^{\prime}(z)\right)^{2}\right) \mid<\infty .
\end{aligned}
$$

Using these facts and the boundedness of the function $\varphi(z)$ again, we get

$$
K_{2}=\sup _{z \in D}\left(1-|z|^{2}\right)^{\alpha}\left|\left(\varphi^{\prime}(z)\right)^{2} u(z)\right|<+\infty .
$$

Fix $a \in D$; we take the test functions

$$
f_{a}(z)=-\frac{1-|a|^{2}}{(1-\bar{a} z)^{1+1 / p}}+2 \frac{\left(1-|a|^{2}\right)^{2}}{(1-\bar{a} z)^{2+1 / p}}-\frac{\left(1-|a|^{2}\right)^{3}}{(1-\bar{a} z)^{3+1 / p}}
$$

for $z \in D$. From Lemma 1 we obtain that $f_{a} \in H^{p}$ and $\sup _{a}\left\|f_{a}\right\|_{p} \leq C(p)<\infty$ with a direct calculation. Since $f_{a}(a)=0, f_{a}^{\prime}(a)=0$, and $f_{a}^{\prime \prime}(a)=-2 \bar{a}^{2} /\left(1-|a|^{2}\right)^{2+1 / p}$, it follows that, for all $\lambda \in D$ with $|\varphi(\lambda)|>1 / 2$, we have

$$
\begin{aligned}
C\left\|f_{a}\right\|_{*} \geq & \left\|u C_{\varphi} f_{a}\right\|_{*} \geq \sup _{z \in D}\left(1-|z|^{2}\right)^{\alpha}\left|\left(u C_{\varphi} f_{a}\right)^{\prime \prime}(z)\right| \\
= & \sup _{z \in D}\left(1-|z|^{2}\right)^{\alpha} \\
& \times \mid\left(2 \varphi^{\prime}(z) u^{\prime}(z)+\varphi^{\prime \prime}(z) u(z)\right) f_{a}^{\prime}(\varphi(z)) \\
& +f_{a}^{\prime \prime}(\varphi(z))\left(\varphi^{\prime}(z)\right)^{2} u(z) \\
& +u^{\prime \prime}(z) f_{a}(\varphi(z)) \mid
\end{aligned}
$$

Let $a=\varphi(\lambda)$; it follows that

$$
\begin{aligned}
C\left\|f_{a}\right\|_{*} \geq\left(1-|\lambda|^{2}\right)^{\alpha} \\
\times \mid \\
\quad\left(2 \varphi^{\prime}(\lambda) u^{\prime}(\lambda)+\varphi^{\prime \prime}(\lambda) u(\lambda)\right) f_{\varphi(\lambda)}^{\prime}(\varphi(\lambda)) \\
\quad+f_{\varphi(\lambda)}^{\prime \prime}(\varphi(\lambda))\left(\varphi^{\prime}(\lambda)\right)^{2} u(\lambda) \\
\quad+u^{\prime \prime}(\lambda) f_{\varphi(\lambda)}(\varphi(\lambda)) \mid
\end{aligned}
$$

$$
\begin{aligned}
& =\left(1-|\lambda|^{2}\right)^{\alpha}\left|\left(\varphi^{\prime}(\lambda)\right)^{2} u(\lambda) \frac{2(\overline{\varphi(\lambda)})^{2}}{\left(1-|\varphi(\lambda)|^{2}\right)^{2+1 / p}}\right| \\
& \geq \frac{1}{2} \frac{\left(1-|\lambda|^{2}\right)^{\alpha}\left|u(\lambda)\left(\varphi^{\prime}(\lambda)\right)^{2}\right|}{\left(1-|\varphi(\lambda)|^{2}\right)^{2+1 / p}} .
\end{aligned}
$$

For all $\lambda \in D$ with $|\varphi(\lambda)| \leq 1 / 2$, by (17), we have

$$
\begin{aligned}
& \sup _{\lambda \in D} \frac{\left(1-|\lambda|^{2}\right)^{\alpha}\left|\mathcal{u}(\lambda)\left(\varphi^{\prime}(\lambda)\right)^{2}\right|}{1-|\varphi(\lambda)|^{1 / p+2}} \\
& \leq\left(\frac{4}{3}\right)^{1 / p+2} \sup _{\lambda \in D}\left(1-|\lambda|^{2}\right)^{\alpha}\left|u(\lambda)\left(\varphi^{\prime}(\lambda)\right)^{2}\right| \\
& \quad<+\infty .
\end{aligned}
$$

Hence (12) holds.

Next, fix $a \in D$; we take another test functions

$$
g_{a}(z)=-3 \frac{1-|a|^{2}}{(1-\bar{a} z)^{1 / p+1}}+5 \frac{\left(1-|a|^{2}\right)^{2}}{(1-\bar{a} z)^{1 / p+2}}-2 \frac{\left(1-|a|^{2}\right)^{3}}{(1-\bar{a} z)^{1 / p+3}}
$$

for $z \in D$. From Lemma 1 we obtain that $g_{a} \in H^{p}$ and $\sup _{a}\left\|g_{a}\right\|_{p} \leq C(p)<\infty$ with a direct calculation. Since $g_{a}(a)=0, g_{a}^{\prime \prime}(a)=0$, and $g_{a}^{\prime}(a)=\bar{a} /\left(1-|a|^{2}\right)^{1 / p+1}$, it follows that, for all $\lambda \in D$ with $|\varphi(\lambda)|>1 / 2$, we obtain that

$$
\begin{aligned}
C\left\|g_{a}\right\|_{*} \geq & \left\|u C_{\varphi} g_{a}(z)\right\|_{*} \\
\geq & \sup _{z \in D}\left(1-|z|^{2}\right)^{\alpha}\left|\left(u C_{\varphi} g_{a}\right)^{\prime \prime}(z)\right| \\
= & \sup _{z \in D}\left(1-|z|^{2}\right)^{\alpha} \\
& \times \mid\left(2 \varphi^{\prime}(z) u^{\prime}(z)+\varphi^{\prime \prime}(z) u(z)\right) g_{a}^{\prime}(\varphi(z)) \\
& \quad+g_{a}^{\prime \prime}(\varphi(z))\left(\varphi^{\prime}(z)\right)^{2} u(z) \\
& \quad+u^{\prime \prime}(z) g_{a}(\varphi(z)) \mid \\
\geq & \left(1-|\lambda|^{2}\right)^{\alpha}
\end{aligned}
$$




$$
\begin{aligned}
& \times \mid\left(2 \varphi^{\prime}(\lambda) u^{\prime}(\lambda)+\varphi^{\prime \prime}(\lambda) u(\lambda)\right) g_{\varphi(\lambda)}^{\prime}(\varphi(\lambda)) \\
&+g_{\varphi(\lambda)}^{\prime \prime}(\varphi(\lambda))\left(\varphi^{\prime}(\lambda)\right)^{2} u(\lambda) \\
&+u^{\prime \prime}(\lambda) g_{\varphi(\lambda)}(\varphi(\lambda)) \mid \\
&=\left(1-|\lambda|^{2}\right)^{\alpha} \\
& \times \mid\left(2 \varphi^{\prime}(\lambda) u^{\prime}(\lambda)+\varphi^{\prime \prime}(\lambda) u(\lambda)\right) \\
& \quad \times \frac{\frac{1}{\varphi(\lambda)}}{\left(1-|\varphi(\lambda)|^{2}\right)^{1 / p+1}} \mid \\
& \geq \frac{1}{2} \frac{\left(1-|\lambda|^{2}\right)^{\alpha}\left|2 \varphi^{\prime}(\lambda) u^{\prime}(\lambda)+\varphi^{\prime \prime}(\lambda) u(\lambda)\right|}{\left(1-|\varphi(\lambda)|^{2}\right)^{1 / p+1}} .
\end{aligned}
$$

For all $\lambda \in D$ with $|\varphi(\lambda)| \leq 1 / 2$, by (15), we have

$$
\begin{aligned}
& \sup _{|\varphi(\lambda)| \leq 1 / 2} \frac{\left(1-|\lambda|^{2}\right)^{\alpha}\left|2 \varphi^{\prime}(\lambda) u^{\prime}(\lambda)+\varphi^{\prime \prime}(\lambda) u(\lambda)\right|}{\left(1-|\varphi(\lambda)|^{2}\right)^{1 / p+1}} \\
& \leq\left(\frac{4}{3}\right)^{1 / p+1} \sup _{|\varphi(\lambda)| \leq 1 / 2}\left(1-|\lambda|^{2}\right)^{\alpha} \\
& \quad \times\left|2 \varphi^{\prime}(\lambda) u^{\prime}(\lambda)+\varphi^{\prime \prime}(\lambda) u(\lambda)\right| \\
&<\infty .
\end{aligned}
$$

Hence (13) holds.

Finally, fix $a \in D$, and, for all $z \in D$, let

$$
\begin{aligned}
h_{a}(z)= & -\frac{p+3}{p+1} \frac{1-|a|^{2}}{(1-\bar{a} z)^{1 / p+1}} \\
& +\frac{2(p+3)}{p+2} \frac{\left(1-|a|^{2}\right)^{2}}{(1-\bar{a} z)^{1 / p+2}}-\frac{\left(1-|a|^{2}\right)^{3}}{(1-\bar{a} z)^{1 / p+3}} .
\end{aligned}
$$

From Lemma 1 we obtain that $h_{a} \in H^{p}$ and $\sup _{a}\left\|h_{a}\right\|_{p} \leq$ $C(p)<\infty$ with a direct calculation. Since $h_{a}^{\prime}(a)=0$, $h_{a}^{\prime \prime}(a)=0$, and $h_{a}(a)=-2 /(p+1)(p+2)\left(1-|a|^{2}\right)^{1 / p}$, it follows that, for all $\lambda \in D$, we obtain that

$$
\begin{aligned}
C\left\|h_{a}\right\|_{*} \geq & \left\|u C_{\varphi} h_{a}(z)\right\|_{*} \\
\geq & \sup _{z \in D}\left(1-|z|^{2}\right)^{\alpha}\left|\left(u C_{\varphi} h_{a}\right)^{\prime \prime}(z)\right| \\
= & \sup _{z \in D}\left(1-|z|^{2}\right)^{\alpha} \\
& \times \mid\left(2 \varphi^{\prime}(z) u^{\prime}(z)+\varphi^{\prime \prime}(z) u(z)\right) h_{a}^{\prime}(\varphi(z)) \\
& \quad+h_{a}^{\prime \prime}(\varphi(z))\left(\varphi^{\prime}(z)\right)^{2} u(z) \\
& \quad+u^{\prime \prime}(z) h_{a}(\varphi(z)) \mid \\
\geq & \left(1-|\lambda|^{2}\right)^{\alpha} \\
& \times \mid\left(2 \varphi^{\prime}(\lambda) u^{\prime}(\lambda)+\varphi^{\prime \prime}(\lambda) u(\lambda)\right) h_{\varphi(\lambda)}^{\prime}(\varphi(\lambda)) \\
& \quad+h_{\varphi(\lambda)}^{\prime \prime}(\varphi(\lambda))\left(\varphi^{\prime}(\lambda)\right)^{2} u(\lambda) \\
& \quad+u^{\prime \prime}(\lambda) h_{\varphi(\lambda)}(\varphi(\lambda)) \mid \\
= & \left(1-|\lambda|^{2}\right)^{\alpha}\left|u^{\prime \prime}(\lambda) h_{\varphi(\lambda)}(\varphi(\lambda))\right| \\
= & \frac{2}{(p+1)(p+2)} \frac{\left(1-|\lambda|^{2}\right)^{\alpha}\left|u^{\prime \prime}(\lambda)\right|}{\left(1-|\varphi(\lambda)|^{2}\right)^{1 / p} .}
\end{aligned}
$$

Then (11) holds.

Conversely, suppose that (11), (12), and (13) hold. For $f \in$ $H^{p}$, by Lemma 2 , we have the following inequality:

$$
\begin{aligned}
\left(1-|z|^{2}\right)^{\alpha} & \left|\left(u C_{\varphi} f\right)^{\prime \prime}(z)\right| \\
= & \left(1-|z|^{2}\right)^{\alpha} \\
& \times \mid\left(2 \varphi^{\prime}(z) u^{\prime}(z)+\varphi^{\prime \prime}(z) u(z)\right) f^{\prime}(\varphi(z)) \\
& +f^{\prime \prime}(\varphi(z))\left(\varphi^{\prime}(z)\right)^{2} u(z) \\
& \quad+u^{\prime \prime}(z) f(\varphi(z)) \mid \\
\leq & \left(1-|z|^{2}\right)^{\alpha} \\
& \times\left|\left(2 \varphi^{\prime}(z) u^{\prime}(z)+\varphi^{\prime \prime}(z) u(z)\right) f^{\prime}(\varphi(z))\right| \\
& +\left(1-|z|^{2}\right)^{\alpha}\left|f^{\prime \prime}(\varphi(z))\left(\varphi^{\prime}(z)\right)^{2} u(z)\right| \\
& +\left(1-|z|^{2}\right)\left|u^{\prime \prime}(z) f(\varphi(z))\right|
\end{aligned}
$$




$$
\begin{aligned}
& \leq \frac{\left(1-|z|^{2}\right)^{\alpha}\left|2 \varphi^{\prime}(z) u^{\prime}(z)+\varphi^{\prime \prime}(z) u(z)\right|}{\left(1-|\varphi(z)|^{2}\right)^{1 / p+1}}\|f\|_{p} \\
& +\frac{\left(1-|z|^{2}\right)^{\alpha}\left|\left(\varphi^{\prime}(z)\right)^{2} u(z)\right|}{\left(1-|\varphi(z)|^{2}\right)^{1 / p+2}}\|f\|_{p} \\
& +\frac{\left(1-|z|^{2}\right)^{\alpha}\left|u^{\prime \prime}(z)\right|}{\left(1-|\varphi(z)|^{2}\right)^{1 / p}}\|f\|_{p} \\
& \leq C\|f\|_{p},
\end{aligned}
$$

$|u(0) f(\varphi(0))|+\left|u^{\prime}(0) f(\varphi(0))\right|+\left|u(0) f^{\prime}(\varphi(0))\right|$

$$
\begin{gathered}
\leq\left(\frac{|u(0)|}{\left(1-|\varphi(0)|^{2}\right)^{1 / p}}+\frac{\left|u^{\prime}(0)\right|}{\left(1-|\varphi(0)|^{2}\right)^{1 / p}}\right. \\
\left.+\frac{|u(0)|}{\left(1-|\varphi(0)|^{2}\right)^{1 / p+1}}\right)\|f\|_{p} \cdot
\end{gathered}
$$

This shows that $u C_{\varphi}$ is bounded. This completes the proof of Theorem 4.

Theorem 5. Let $\alpha>0,0<p<\infty$, and $u$ be an analytic function on the unit disc $D$ and $\varphi$ an analytic self-map of $D$. Then $u C_{\varphi}: H^{p} \rightarrow \mathscr{X}_{\alpha, 0}$ is a bounded operator provided that the following are satisfied:

$$
\begin{gathered}
\lim _{|z| \rightarrow 1^{-}} \frac{\left(1-|z|^{2}\right)^{\alpha}\left|u^{\prime \prime}(z)\right|}{\left(1-|\varphi(z)|^{2}\right)^{1 / p}}=0, \\
\lim _{|z| \rightarrow 1^{-}} \frac{\left(1-|z|^{2}\right)^{\alpha}\left|u(z)\left(\varphi^{\prime}(z)\right)^{2}\right|}{\left(1-|\varphi(z)|^{2}\right)^{1 / p+2}}=0, \\
\lim _{|z| \rightarrow 1^{-}} \frac{\left(1-|z|^{2}\right)^{\alpha}\left|2 \varphi^{\prime}(z) u^{\prime}(z)+\varphi^{\prime \prime}(z) u(z)\right|}{\left(1-|\varphi(z)|^{2}\right)^{1 / p+1}}=0 .
\end{gathered}
$$

Conversely, if $u C_{\varphi}: H^{p} \rightarrow \mathscr{Z}_{\alpha, 0}$ is a bounded operator, then $u \in \mathscr{Z}_{\alpha, 0}$, (11), (12), and (13) hold, and the following are satisfied:

$$
\begin{gathered}
\lim _{|z| \rightarrow 1^{-}}\left(1-|z|^{2}\right)^{\alpha}\left|u(z)\left(\varphi^{\prime}(z)\right)^{2}\right|=0, \\
\lim _{|z| \rightarrow 1^{-}}\left(1-|z|^{2}\right)^{\alpha}\left|2 \varphi^{\prime}(z) u^{\prime}(z)+\varphi^{\prime \prime}(z) u(z)\right|=0 .
\end{gathered}
$$

Proof. Assume that (28), (29), and (30) hold. Then for any $\epsilon>$ 0 , there is a constant $\delta, 0<\delta<1$, such that $\delta<|z|<1$ implies

$$
\begin{gathered}
\frac{\left(1-|z|^{2}\right)^{\alpha}\left|u(z)\left(\varphi^{\prime}(z)\right)^{2}\right|}{\left(1-|\varphi(z)|^{2}\right)^{1 / p+2}}<\epsilon, \\
\frac{\left(1-|z|^{2}\right)^{\alpha}\left|u^{\prime \prime}(z)\right|}{\left(1-|\varphi(z)|^{2}\right)^{1 / p}}<\epsilon, \\
\frac{\left(1-|z|^{2}\right)^{\alpha}\left|2 \varphi^{\prime}(z) u^{\prime}(z)+\varphi^{\prime \prime}(z) u(z)\right|}{\left(1-|\varphi(z)|^{2}\right)^{1 / p+1}}<\epsilon .
\end{gathered}
$$

Then, for any $f \in H^{p}$, from Lemma 2 we obtain that

$$
\begin{aligned}
\left(1-|z|^{2}\right)^{\alpha} & \left|\left(u C_{\varphi} f\right)^{\prime \prime}(z)\right| \\
= & \left(1-|z|^{2}\right)^{\alpha} \\
& \times \mid\left(2 \varphi^{\prime}(z) u^{\prime}(z)+\varphi^{\prime \prime}(z) u(z)\right) f^{\prime}(\varphi(z)) \\
& +f^{\prime \prime}(\varphi(z))\left(\varphi^{\prime}(z)\right)^{2} u(z) \\
& \quad+u^{\prime \prime}(z) f(\varphi(z)) \mid \\
\leq & \left(1-|z|^{2}\right)^{\alpha}\left|2 \varphi^{\prime}(z) u^{\prime}(z)+\varphi^{\prime \prime}(z) u(z)\right| \\
& \times \frac{1}{\left(1-|\varphi(z)|^{2}\right)^{1 / p+1}}\|f\|_{p} \\
+ & \left(1-|z|^{2}\right)^{\alpha}\left|\left(\varphi^{\prime}(z)\right)^{2} u(z)\right| \frac{1}{\left(1-|\varphi(z)|^{2}\right)^{1 / p+2}}\|f\|_{p} \\
& +\frac{\left(1-|z|^{2}\right)\left|u^{\prime \prime}(z)\right|}{\left(1-|\varphi(z)|^{2}\right)^{1 / p}\|f\|_{p} \leq 3\|f\|_{p} \epsilon .}
\end{aligned}
$$

Hence $u C_{\varphi} f \in \mathscr{Z}_{\alpha, 0}$ for all $f \in \mathscr{Z}_{\alpha, 0}$. On the other hand, (25), (28), and (29) imply that (11), (12), and (13) hold; then $u C_{\varphi}: H^{p} \rightarrow \mathscr{Z}_{\alpha}$ is bounded by Theorem 4 . So $u C_{\varphi}: H^{p} \rightarrow$ $\mathscr{Z}_{\alpha, 0}$ is bounded.

Conversely, assume that $u C_{\varphi}$ is bounded from $H^{p}$ to the little Zygmund type space $\mathscr{Z}_{\alpha, 0}$. Then $u=u C_{\varphi} 1 \in \mathscr{Z}_{\alpha, 0}$. Also $u \varphi=u C_{\varphi} z \in \mathscr{E}_{\alpha, 0}$; thus

$$
\begin{aligned}
&\left(1-|z|^{2}\right)^{\alpha}\left|2 \varphi^{\prime}(z) u^{\prime}(z)+\varphi^{\prime \prime}(z) u(z)+\varphi(z) u^{\prime \prime}(z)\right| \longrightarrow 0 \\
&\left(|z| \longrightarrow 1^{-}\right) .
\end{aligned}
$$

Since $|\varphi| \leq 1$ and $u \in \mathscr{Z}_{\alpha, 0}$, we have $\lim _{|z| \rightarrow 1^{-}}(1-$ $\left.|z|^{2}\right)^{\alpha}\left|2 \varphi^{\prime}(z) u^{\prime}(z)+\varphi^{\prime \prime}(z) u(z)\right|=0$. Hence (32) holds. 
Similarly, $u C_{\varphi} z^{2} \in \mathscr{Z}_{\alpha, 0}$; then

$$
\begin{aligned}
& \left(1-|z|^{2}\right)^{\alpha} \\
& \times \mid 4 \varphi(z) \varphi^{\prime}(z) u^{\prime}(z)+\varphi^{2}(z) u^{\prime \prime}(z) \\
& +2 u(z)\left(\varphi(z) \varphi^{\prime \prime}(z)+\left(\varphi^{\prime}(z)\right)^{2}\right) \mid \longrightarrow 0 \\
& \left(|z| \longrightarrow 1^{-}\right) .
\end{aligned}
$$

By (32), $|\varphi| \leq 1$, and $u \in \mathscr{Z}_{\alpha, 0}$, we get that $\lim _{|z| \rightarrow 1^{-}}(1-$ $\left.|z|^{2}\right)^{\alpha}\left|u(z)\left(\varphi^{\prime}(z)\right)^{2}\right|=0$; that is, (31) holds.

On the other hand, from Lemma 3 and Theorem 4, we obtain that (11), (12), and (13) hold.

\section{Compactness of $u C_{\varphi}$}

In order to prove the compactness of $u C_{\varphi}$ from $H^{p}$ to the Zygmund spaces $\mathscr{Z}_{\alpha}$, we require the following lemmas.

Lemma 6. Let $\alpha>0,0<p<\infty$, and $u$ be an analytic function on the unit disc $D$ and $\varphi$ an analytic self-map of $D$. Suppose that $u C_{\varphi}$ is a bounded operator from $H^{p}$ to $\mathscr{Z}_{\alpha}$. Then $u C_{\varphi}$ is compact if and only if, for any bounded sequence $\left\{f_{n}\right\}$ in $H^{p}$ which converges to 0 uniformly on compact subsets of $D$, one has $\left\|u C_{\varphi}\left(f_{n}\right)\right\|_{*} \rightarrow 0$ as $n \rightarrow \infty$.

The proof is similar to that of Proposition 3.11 in [32]. The details are omitted.

Theorem 7. Let $\alpha>0,0<p<\infty, u$ be an analytic function on the unit disc $D$ and $\varphi$ an analytic self-map of $D$. Then $u C_{\varphi}$ is a compact operator from $H^{p}$ to $\mathscr{Z}_{\alpha}$ if and only if $u C_{\varphi}$ is a bounded operator and the following are satisfied:

(i) $\lim _{|\varphi(z)| \rightarrow 1^{-}} \frac{\left(1-|z|^{2}\right)^{\alpha}\left|u^{\prime \prime}(z)\right|}{\left(1-|\varphi(z)|^{2}\right)^{1 / p}}=0$,

(ii) $\lim _{|\varphi(z)| \rightarrow 1^{-}} \frac{\left(1-|z|^{2}\right)^{\alpha}\left|u(z)\left(\varphi^{\prime}(z)\right)^{2}\right|}{\left(1-|\varphi(z)|^{2}\right)^{1 / p+2}}=0$,

(iii) $\lim _{|\varphi(z)| \rightarrow 1^{-}} \frac{\left(1-|z|^{2}\right)^{\alpha}\left|2 \varphi^{\prime}(z) u^{\prime}(z)+\varphi^{\prime \prime}(z) u(z)\right|}{\left(1-|\varphi(z)|^{2}\right)^{1 / p+1}}=0$.

Proof. Suppose that $u C_{\varphi}$ is compact from $H^{p}$ to the Zygmund type space $\mathscr{Z}_{\alpha}$. Let $\left\{z_{n}\right\}$ be a sequence in $D$ such that $\left|\varphi\left(z_{n}\right)\right| \rightarrow 1$ as $n \rightarrow \infty$. If such a sequence does not exist, then (37) are automatically satisfied. Without loss of generality we may suppose that $\left|\varphi\left(z_{n}\right)\right|>1 / 2$ for all $n$. We take the test functions

$$
\begin{aligned}
f_{n}(z)= & -\frac{1-\left|\varphi\left(z_{n}\right)\right|^{2}}{\left(1-\overline{\varphi\left(z_{n}\right)} z\right)^{1 / p+1}}+2 \frac{\left(1-\left|\varphi\left(z_{n}\right)\right|^{2}\right)^{2}}{\left(1-\overline{\varphi\left(z_{n}\right) z}\right)^{1 / p+2}} \\
& -\frac{\left(1-\left|\varphi\left(z_{n}\right)\right|^{2}\right)^{3}}{\left(1-\overline{\varphi\left(z_{n}\right)} z\right)^{1 / p+3}} .
\end{aligned}
$$

By a direct calculation, we may easily prove that $\left\{f_{n}\right\}$ converges to 0 uniformly on compact subsets of $D$ and $\sup _{n}\left\|f_{n}\right\|_{p} \leq C(p)<\infty$. Then $\left\{f_{n}\right\}$ is a bounded sequence in $H^{p}$ which converges to 0 uniformly on compact subsets of $D$. Then $\lim _{n \rightarrow \infty}\left\|u C_{\varphi}\left(f_{n}\right)\right\|_{*}=0$ by Lemma 6 . Note that

$$
\begin{aligned}
& f_{n}\left(\varphi\left(z_{n}\right)\right)=0, \quad f_{n}^{\prime}\left(\varphi\left(z_{n}\right)\right)=0, \\
& f_{n}^{\prime \prime}\left(\varphi\left(z_{n}\right)\right)=-\frac{2{\overline{\varphi\left(z_{n}\right)}}^{2}}{\left(1-\left|\varphi\left(z_{n}\right)\right|^{2}\right)^{1 / p+2}} .
\end{aligned}
$$

It follows that

$$
\begin{aligned}
\left\|u C_{\varphi} f_{n}\right\|_{*} \geq & \left\|u C_{\varphi} f_{n}\right\|_{\mathscr{Z}_{\alpha}} \\
\geq & \left(1-\left|z_{n}\right|^{2}\right)^{\alpha} \\
& \times \mid\left(2 u^{\prime}\left(z_{n}\right) \varphi^{\prime}\left(z_{n}\right)+\varphi^{\prime \prime}\left(z_{n}\right) u\left(z_{n}\right)\right) \\
& \quad \times f_{n}^{\prime}\left(\varphi\left(z_{n}\right)\right)+u\left(z_{n}\right) f_{n}^{\prime \prime}\left(\varphi\left(z_{n}\right)\right)\left(\varphi^{\prime}\left(z_{n}\right)\right)^{2} \\
& \quad+u^{\prime \prime}\left(z_{n}\right) f_{n}\left(\varphi\left(z_{n}\right)\right) \mid \\
= & \left.\quad+\mid\left(\left.z_{n}\right|^{2}\right)^{\alpha}\left(z_{n}\right)\right)^{2} u\left(z_{n}\right) \frac{2\left(z_{n}\right)^{2}}{\left(1-\left|\varphi\left(z_{n}\right)\right|^{2}\right)^{1 / p+2}} \mid .
\end{aligned}
$$

Then

$$
\lim _{n \rightarrow \infty} \frac{\left(1-\left|z_{n}\right|^{2}\right)^{\alpha}\left|u\left(z_{n}\right)\left(\varphi^{\prime}\left(z_{n}\right)\right)^{2}\right|}{\left(1-\left|\varphi\left(z_{n}\right)\right|^{2}\right)^{1 / p+2}}=0 .
$$

Next, let

$$
\begin{aligned}
g_{n}(z)= & -3 \frac{1-\left|\varphi\left(z_{n}\right)\right|^{2}}{\left(1-\overline{\varphi\left(z_{n}\right)} z\right)^{1 / p+1}}+5 \frac{\left(1-\left|\varphi\left(z_{n}\right)\right|^{2}\right)^{2}}{\left(1-\overline{\varphi\left(z_{n}\right)} z\right)^{1 / p+2}} \\
& -2 \frac{\left(1-\left|\varphi\left(z_{n}\right)\right|^{2}\right)^{3}}{\left(1-\overline{\varphi\left(z_{n}\right)} z\right)^{1 / p+3}} .
\end{aligned}
$$


By a direct calculation we obtain that $g_{n} \rightrightarrows 0(n \rightarrow \infty)$ on compact subsets of $D$ and $\sup _{n}\left\|g_{n}\right\|_{p} \leq C(p)<+\infty$. Consequently, $\left\{g_{n}\right\}$ is a bounded sequence in $H^{p}$ which converges to 0 uniformly on compact subsets of $D$. Then $\lim _{n \rightarrow \infty}\left\|u C_{\varphi}\left(g_{n}\right)\right\|_{*}=0$ by Lemma 6 . Note that $g_{n}\left(\varphi\left(z_{n}\right)\right) \equiv$ $0, g_{n}^{\prime \prime}\left(\varphi\left(z_{n}\right)\right) \equiv 0$ and $g_{n}^{\prime}\left(\varphi\left(z_{n}\right)\right)=\overline{\varphi\left(z_{n}\right)} /\left(1-\left|\varphi\left(z_{n}\right)\right|^{2}\right)^{1 / p+1}$; it follows that

$$
\begin{aligned}
& \left\|u C_{\varphi} g_{n}\right\|_{*} \geq\left\|u C_{\varphi} g_{n}\right\|_{\mathscr{Z}_{\alpha}} \\
& \geq\left(1-\left|z_{n}\right|^{2}\right)^{\alpha} \\
& \times \mid\left(2 u^{\prime}\left(z_{n}\right) \varphi^{\prime}\left(z_{n}\right)+\varphi^{\prime \prime}\left(z_{n}\right) u\left(z_{n}\right)\right) \\
& \times g_{n}^{\prime}\left(\varphi\left(z_{n}\right)\right)+u\left(z_{n}\right) g_{n}^{\prime \prime}\left(\varphi\left(z_{n}\right)\right)\left(\varphi^{\prime}\left(z_{n}\right)\right)^{2} \\
& +u^{\prime \prime}\left(z_{n}\right) g_{n}\left(\varphi\left(z_{n}\right)\right) \\
& =\left(1-\left|z_{n}\right|^{2}\right)^{\alpha} \\
& \times \mid\left(2 u^{\prime}\left(z_{n}\right) \varphi^{\prime}\left(z_{n}\right)+\varphi^{\prime \prime}\left(z_{n}\right) u\left(z_{n}\right)\right) \\
& \times \frac{\overline{\varphi\left(z_{n}\right)}}{\left(1-\left|\overline{\varphi\left(z_{n}\right)}\right|^{2}\right)^{1 / p+1}} \mid
\end{aligned}
$$

Then $\lim _{n \rightarrow \infty}\left(1-\left|z_{n}\right|^{2}\right)^{\alpha}\left(2 u^{\prime}\left(z_{n}\right) \varphi^{\prime}\left(z_{n}\right)+\varphi^{\prime \prime}\left(z_{n}\right) u\left(z_{n}\right)\right) /(1-$ $\left.\left|\varphi\left(z_{n}\right)\right|^{2}\right)^{1 / p+1}=0$.

Finally, let

$$
\begin{aligned}
h_{n}(z)= & -\frac{p+3}{p+1} \frac{1-\left|\varphi\left(z_{n}\right)\right|^{2}}{\left(1-\overline{\varphi\left(z_{n}\right)} z\right)^{1 / p+1}} \\
& +\frac{2(p+3)}{p+2} \frac{\left(1-\left|\varphi\left(z_{n}\right)\right|^{2}\right)^{2}}{\left(1-\overline{\varphi\left(z_{n}\right)} z\right)^{1 / p+2}} \\
& -\frac{\left(1-\left|\varphi\left(z_{n}\right)\right|^{2}\right)^{3}}{\left(1-\overline{\varphi\left(z_{n}\right) z}\right)^{1 / p+3}} .
\end{aligned}
$$

By a direct calculation we obtain that $h_{n} \rightrightarrows 0(n \rightarrow \infty)$ on compact subsets of $D$ and $\sup _{n}\left\|h_{n}\right\|_{p}<\infty$. Consequently, $\left\{h_{n}\right\}$ is a bounded sequence in $H^{p}$ which converges to 0 uniformly on compact subsets of $D$. Then $\lim _{n \rightarrow \infty}\left\|u C_{\varphi}\left(h_{n}\right)\right\|_{*}=0$ by Lemma 6 . Note that $h_{n}\left(\varphi\left(z_{n}\right)\right)=2 /(p+1)(p+2)\left(1-\left|\varphi\left(z_{n}\right)\right|^{2}\right)^{1 / p}, h_{n}^{\prime}\left(\varphi\left(z_{n}\right)\right) \equiv 0$, and $h_{n}^{\prime \prime}\left(\varphi\left(z_{n}\right)\right) \equiv 0$; it follows that

$$
\begin{aligned}
\left\|u C_{\varphi} h_{n}\right\|_{*} \geq & \left\|u C_{\varphi} h_{n}\right\|_{\mathscr{Z}_{\alpha}} \\
\geq & \left(1-\left|z_{n}\right|^{2}\right)^{\alpha} \\
& \times \mid\left(2 u^{\prime}\left(z_{n}\right) \varphi^{\prime}\left(z_{n}\right)+\varphi^{\prime \prime}\left(z_{n}\right) u\left(z_{n}\right)\right) \\
& \times h_{n}^{\prime}\left(\varphi\left(z_{n}\right)\right)+u\left(z_{n}\right) h_{n}^{\prime \prime}\left(\varphi\left(z_{n}\right)\right)\left(\varphi^{\prime}\left(z_{n}\right)\right)^{2} \\
& \quad+u^{\prime \prime}\left(z_{n}\right) h_{n}\left(\varphi\left(z_{n}\right)\right) \mid \\
= & \frac{2\left(1-\left|z_{n}\right|^{2}\right)^{\alpha}\left|u^{\prime \prime}\left(z_{n}\right)\right|}{(p+1)(p+2)\left(1-\left|\varphi\left(z_{n}\right)\right|^{2}\right)^{1 / p}} .
\end{aligned}
$$

Then $\lim _{n \rightarrow \infty}\left(\left(1-\left|z_{n}\right|^{2}\right)^{\alpha}\left|u^{\prime \prime}\left(z_{n}\right)\right| /\left(1-\left|\varphi\left(z_{n}\right)\right|^{2}\right)^{1 / p}\right)=0$. The proof of the necessary is completed.

Conversely, Suppose that (37) hold. Since $u C_{\varphi}$ is a bounded operator, from Theorem 4 , we have

$$
\begin{gathered}
M_{1} \triangleq \sup _{z \in D}\left(1-|z|^{2}\right)^{\alpha}\left|u(z)\left(\varphi^{\prime}(z)\right)^{2}\right|<\infty, \\
M_{2} \triangleq \sup _{z \in D}\left(1-|z|^{2}\right)^{\alpha}\left|u^{\prime \prime}(z)\right|<\infty, \\
M_{3} \triangleq \sup _{z \in D}\left(1-|z|^{2}\right)^{\alpha}\left|2 \varphi^{\prime}(z) u^{\prime}(z)+\varphi^{\prime \prime}(z) u(z)\right|<\infty .
\end{gathered}
$$

Let $\left\{f_{n}\right\}$ be a bounded sequence in $H^{p}$ with $\left\|f_{n}\right\|_{p} \leq 1$ and $f_{n} \rightarrow 0$ uniformly on compact subsets of $D$. We only prove $\lim _{n \rightarrow \infty}\left\|u C_{\varphi}\left(f_{n}\right)\right\|_{*}=0$ by Lemma 6 . By the assumption, for any $\epsilon>0$, there is a constant $\delta, 0<\delta<1$, such that $\delta<|\varphi(z)|<1$ implies

$$
\begin{gathered}
\frac{\left(1-|z|^{2}\right)^{\alpha}\left|u(z)\left(\varphi^{\prime}(z)\right)^{2}\right|}{\left(1-|\varphi(z)|^{2}\right)^{1 / p+2}}<\epsilon, \\
\frac{\left(1-|z|^{2}\right)^{\alpha}\left|u^{\prime \prime}(z)\right|}{\left(1-|\varphi(z)|^{2}\right)^{1 / p}}<\epsilon, \\
\frac{\left(1-|z|^{2}\right)^{\alpha}\left|2 \varphi^{\prime}(z) u^{\prime}(z)+\varphi^{\prime \prime}(z) u(z)\right|}{\left(1-|\varphi(z)|^{2}\right)^{1 / p+1}}<\epsilon .
\end{gathered}
$$


Let $K=\{w \in D:|w| \leq \delta\}$. Note that $K$ is a compact subset of $D$. Then from Lemma 2 it follows that

$$
\begin{aligned}
\left\|u C_{\varphi} f_{n}\right\|_{\mathscr{I}_{\alpha}}= & \sup _{z \in D}\left(1-|z|^{2}\right)^{\alpha}\left|\left(u C_{\varphi} f_{n}\right)^{\prime \prime}(z)\right| \\
= & \left(1-|z|^{2}\right)^{\alpha} \\
& \times \mid\left(2 \varphi^{\prime}(z) u^{\prime}(z)+\varphi^{\prime \prime}(z) u(z)\right) f_{n}^{\prime}(\varphi(z)) \\
& +f_{n}^{\prime \prime}(\varphi(z))\left(\varphi^{\prime}(z)\right)^{2} u(z) \\
& +u^{\prime \prime}(z) f_{n}(\varphi(z)) \mid \\
\leq & \sup _{z \in D}\left(1-|z|^{2}\right)^{\alpha} \\
& \times\left|\left(2 \varphi^{\prime}(z) u^{\prime}(z)+\varphi^{\prime \prime}(z) u(z)\right) f_{n}^{\prime}(\varphi(z))\right| \\
& +\sup _{z \in D}\left(1-|z|^{2}\right)^{\alpha}\left|f_{n}^{\prime \prime}(\varphi(z))\left(\varphi^{\prime}(z)\right)^{2} u(z)\right| \\
+ & \sup _{z \in D}\left(1-|z|^{2}\right)\left|u^{\prime \prime}(z) f_{n}(\varphi(z))\right| \\
\leq & 3 \epsilon+\sup _{|\varphi(z)| \leq \delta}\left(1-|z|^{2}\right)^{\alpha} \\
& \times\left|\left(2 \varphi^{\prime}(z) u^{\prime}(z)+\varphi^{\prime \prime}(z) u(z)\right) f_{n}^{\prime}(\varphi(z))\right| \\
& +\sup _{|\varphi(z)| \leq \delta}\left(1-|z|^{2}\right)^{\alpha}\left|f_{n}^{\prime \prime}(\varphi(z))\left(\varphi^{\prime}(z)\right)^{2} u(z)\right| \\
& +\sup _{|\varphi(z)| \leq \delta}\left(1-|z|^{2}\right)^{\alpha}\left|u^{\prime \prime}(z) f_{n}(\varphi(z))\right| \\
\leq & 3 \epsilon+M_{3} \sup _{w \in K}\left|f_{n}^{\prime}(w)\right|+M_{1} \sup _{w \in K}\left|f_{n}^{\prime \prime}(w)\right| \\
& +M_{2} \sup _{w \in K}\left|f_{n}(w)\right| . \\
&
\end{aligned}
$$

As $n \rightarrow \infty$,

$$
\left\|u C_{\varphi} f_{n}\right\|_{\mathscr{E}_{\alpha}} \longrightarrow 0 .
$$

Hence $u C_{\varphi}$ is compact. This completes the proof of Theorem 7.

In order to prove the compactness of $u C_{\varphi}$ on the little Zygmund spaces $\mathscr{E}_{\alpha, 0}$, we require the following lemma.

Lemma 8. Let $U \subset \mathscr{Z}_{\alpha, 0}$. Then $U$ is compact if and only if it is closed, bounded and satisfies

$$
\lim _{|z| \rightarrow 1^{-}} \sup _{f \in U}\left(1-|z|^{2}\right)\left|f^{\prime \prime}(z)\right|=0 .
$$

The proof is similar to that of Lemma 1 in [1], but we omit it.

Theorem 9. Let $\alpha>0,0<p<\infty, u$ be an analytic function on the unit disc $D$ and $\varphi$ an analytic self-map of $D$. Then $u C_{\varphi}$ is compact from $H^{p}$ to the little Zygmund type spaces $\mathscr{Z}_{\alpha, 0}$ if and only if (28), (29), and (30) hold.

Proof. Assume that (28), (29), and (30) hold. By Theorem 5, we know that $u C_{\varphi}$ is bounded from $H^{p}$ to the little Zygmund type spaces $\mathscr{E}_{\alpha, 0}$. Suppose that $f \in H^{p}$ with $\|f\|_{p} \leq 1$. From Lemmas 1 and 2 we obtain that

$$
\begin{aligned}
\left(1-|z|^{2}\right)^{\alpha}\left|\left(u C_{\varphi} f\right)^{\prime \prime}(z)\right| & \\
\leq & \left(1-|z|^{2}\right)^{\alpha}\left|\left(2 \varphi^{\prime}(z) u^{\prime}(z)+\varphi^{\prime \prime}(z) u(z)\right) f^{\prime}(\varphi(z))\right| \\
& +\left(1-|z|^{2}\right)^{\alpha}\left|f^{\prime \prime}(\varphi(z))\left(\varphi^{\prime}(z)\right)^{2} u(z)\right| \\
& +\left(1-|z|^{2}\right)\left|u^{\prime \prime}(z) f(\varphi(z))\right| \\
\leq & \left(1-|z|^{2}\right)^{\alpha}\left|2 \varphi^{\prime}(z) u^{\prime}(z)+\varphi^{\prime \prime}(z) u(z)\right| \\
& \times \frac{1}{\left(1-|\varphi(z)|^{2}\right)^{1 / p+1}}\|f\|_{p} \\
& +\frac{\left(1-|z|^{2}\right)^{\alpha}\left|\left(\varphi^{\prime}(z)\right)^{2} u(z)\right|}{\left(1-|\varphi(z)|^{2}\right)^{1 / p+2}}\|f\|_{p} \\
& +\frac{\left(1-|z|^{2}\right)^{\alpha}\left|u^{\prime \prime}(z)\right|}{\left(1-|\varphi(z)|^{2}\right)^{1 / p}}\|f\|_{p}
\end{aligned}
$$

thus

$$
\begin{aligned}
\sup & \left\{\left|\left(1-|z|^{2}\right)^{\alpha}\left(u C_{\varphi} f\right)^{\prime \prime}(z)\right|: f \in H^{p},\|f\|_{p} \leq 1\right\} \\
\leq & \left(1-|z|^{2}\right)^{\alpha}\left|2 \varphi^{\prime}(z) u^{\prime}(z)+\varphi^{\prime \prime}(z) u(z)\right| \frac{1}{1-|\varphi(z)|^{2}} \\
& +\frac{\left(1-|z|^{2}\right)^{\alpha}\left|\left(\varphi^{\prime}(z)\right)^{2} u(z)\right|}{\left(1-|\varphi(z)|^{2}\right)^{1 / p+2}}+\frac{\left(1-|z|^{2}\right)^{\alpha}\left|u^{\prime \prime}(z)\right|}{\left(1-|\varphi(z)|^{2}\right)^{1 / p}}
\end{aligned}
$$

and it follows that

$$
\begin{aligned}
& \lim _{|z| \rightarrow 1^{-}} \sup \left\{\left|\left(1-|z|^{2}\right)^{\alpha}\left(u C_{\varphi} f\right)^{\prime \prime}(z)\right|: f \in H^{p},\|f\|_{p} \leq 1\right\} \\
& \quad=0 .
\end{aligned}
$$

Hence $u C_{\varphi}: H^{p} \rightarrow \mathscr{E}_{\alpha, 0}$ is compact by Lemma 8 .

Conversely, suppose that $u C_{\varphi}: H^{p} \rightarrow \mathscr{E}_{\alpha, 0}$ is compact.

Firstly, it is obvious $u C_{\varphi}$ is bounded, from Theorem 5 we have $u \in \mathscr{Z}_{\alpha, 0}$, and (31), (32) hold. On the other hand, we have 


$$
\begin{aligned}
& \lim _{|z| \rightarrow 1^{-}} \sup \left\{\left|\left(1-|z|^{2}\right)^{\alpha}\left(u C_{\varphi} f\right)^{\prime \prime}(z)\right|: f \in H^{p},\|f\|_{p} \leq M\right\} \\
& \quad=0
\end{aligned}
$$

for some $M>0$ by Lemma 6 .

Next, note that the proof of Theorem 4 and the fact that the functions given in (18) are in $H^{p}$ and have norms bounded independently of $a$; we obtain that

$$
\lim _{|z| \rightarrow 1^{-}} \frac{\left(1-|z|^{2}\right)^{\alpha}\left|u(z)\left(\varphi^{\prime}(z)\right)^{2}\right|}{\left(1-|\varphi(z)|^{2}\right)^{1 / p+2}}=0
$$

for $|\varphi(z)|>1 / 2$. However, if $|\varphi(z)| \leq 1 / 2$, by (31), we easily have

$$
\begin{aligned}
& \lim _{|z| \rightarrow 1^{-}} \frac{\left(1-|z|^{2}\right)^{\alpha}\left|u(z)\left(\varphi^{\prime}(z)\right)^{2}\right|}{\left(1-|\varphi(z)|^{2}\right)^{1 / p+2}} \\
& \quad \leq \lim _{|z| \rightarrow 1^{-}}\left(\frac{4}{3}\right)^{1 / p+2}\left(1-|z|^{2}\right)^{\alpha}\left|u(z)\left(\varphi^{\prime}(z)\right)^{2}\right| \\
& \quad=0 .
\end{aligned}
$$

Similarly, note that the functions given in (22) and (25) are in $H^{p}$ and have norms bounded independently of $a$, we obtain that

$$
\begin{gathered}
\lim _{|z| \rightarrow 1^{-}} \frac{\left(1-|z|^{2}\right)^{\alpha}\left|2 \varphi^{\prime}(z) u^{\prime}(z)+\varphi^{\prime \prime}(z) u(z)\right|}{\left(1-|\varphi(z)|^{2}\right)^{1 / p+1}}=0, \\
\lim _{|z| \rightarrow 1^{-}} \frac{\left(1-|z|^{2}\right)^{\alpha}\left|u^{\prime \prime}(z)\right|}{\left(1-|\varphi(z)|^{2}\right)^{1 / p}}=0
\end{gathered}
$$

for $|\varphi(z)|>1 / 2$. However, if $|\varphi(z)| \leq 1 / 2$, from $u \in \mathscr{Z}_{\alpha, 0}$ and (32), we easily have

$$
\begin{aligned}
\lim _{|z| \rightarrow 1^{-}} \frac{\left(1-|z|^{2}\right)^{\alpha}\left|2 \varphi^{\prime}(z) u^{\prime}(z)+\varphi^{\prime \prime}(z) u(z)\right|}{\left(1-|\varphi(z)|^{2}\right)^{1 / p+1}} \\
\leq\left(\frac{4}{3}\right)^{1 / p+1} \lim _{|z| \rightarrow 1^{-}}\left(1-|z|^{2}\right)^{\alpha} \\
\quad \times\left|2 \varphi^{\prime}(z) u^{\prime}(z)+\varphi^{\prime \prime}(z) u(z)\right| \\
=0, \\
\lim _{|z| \rightarrow 1^{-}} \frac{\left(1-|z|^{2}\right)^{\alpha}\left|u^{\prime \prime}(z)\right|}{\left(1-|\varphi(z)|^{2}\right)^{1 / p}} \\
\leq \lim _{|z| \rightarrow 1^{-}}\left(\frac{4}{3}\right)^{1 / p}\left(1-|z|^{2}\right)^{\alpha}\left|u^{\prime \prime}(z)\right| \\
=0 .
\end{aligned}
$$

This completes the proof of Theorem 9.
Remark 10. From Theorems 5 and 9, we conjecture that $u C_{\varphi}$ : $H^{p} \rightarrow \mathscr{Z}_{\alpha, 0}$ is compact if and only if $u C_{\varphi}: H^{p} \rightarrow \mathscr{Z}_{\alpha, 0}$ is bounded.

Taking $u(z)=1$ from Theorems 4, 7, and 9, we obtain the following results about the characterization of the boundedness and compactness of the composition operator $C_{\varphi}: H^{p} \rightarrow \mathscr{E}_{\alpha}\left(\right.$ or $\left.\mathscr{Z}_{\alpha, 0}\right)$.

Corollary 11. Let $\alpha>0,0<p<\infty$, and $\varphi$ be an analytic self-map of $D$. Then $C_{\varphi}: H^{p} \rightarrow \mathscr{Z}_{\alpha}$ is a bounded operator if and only if the following are satisfied:

$$
\begin{gathered}
\sup _{z \in D} \frac{\left(1-|z|^{2}\right)^{\alpha}\left|\left(\varphi^{\prime}(z)\right)^{2}\right|}{\left(1-|\varphi(z)|^{2}\right)^{1 / p+2}}<\infty, \\
\sup _{z \in D} \frac{\left(1-|z|^{2}\right)^{\alpha}\left|\varphi^{\prime \prime}(z)\right|}{\left(1-|\varphi(z)|^{2}\right)^{1 / p+1}}<\infty .
\end{gathered}
$$

Corollary 12. Let $\alpha>0,0<p<\infty$, and $\varphi$ be an analytic self-map of $D$. Then $C_{\varphi}: H^{p} \rightarrow \mathscr{Z}_{\alpha}$ is a compact operator if and only if $C_{\varphi}$ is bounded and the following are satisfied:

$$
\begin{gathered}
\lim _{|\varphi(z)| \rightarrow 1^{-}} \frac{\left(1-|z|^{2}\right)^{\alpha}\left|\left(\varphi^{\prime}(z)\right)^{2}\right|}{\left(1-|\varphi(z)|^{2}\right)^{1 / p+2}}=0, \\
\lim _{|\varphi(z)| \rightarrow 1^{-}} \frac{\left(1-|z|^{2}\right)^{\alpha}\left|\varphi^{\prime \prime}(z)\right|}{\left(1-|\varphi(z)|^{2}\right)^{1 / p+1}}=0 .
\end{gathered}
$$

Corollary 13. Let $\alpha>0,0<p<\infty$, and $\varphi$ be an analytic self-map of $D$. Then $C_{\varphi}: H^{p} \rightarrow \mathscr{Z}_{\alpha, 0}$ is a compact operator if and only if

$$
\begin{aligned}
& \lim _{|z| \rightarrow 1^{-}} \frac{\left(1-|z|^{2}\right)^{\alpha}\left|\left(\varphi^{\prime}(z)\right)^{2}\right|}{\left(1-|\varphi(z)|^{2}\right)^{1 / p+2}}=0 \\
& \lim _{|z| \rightarrow 1^{-}} \frac{\left(1-|z|^{2}\right)^{\alpha}\left|\varphi^{\prime \prime}(z)\right|}{\left(1-|\varphi(z)|^{2}\right)^{1 / p+1}}=0 .
\end{aligned}
$$

In the formulation of corollary, we use the notation $M_{u}$ on $H(D)$ defined by $M_{u} f=u f$ for $f \in H(D)$. Taking $\varphi(z)=z$ from Theorems 4, 5, 7, and 9, we obtain the following results about the characterization of the boundedness and compactness of pointwise multiplier $M_{u}: H^{p} \rightarrow \mathscr{Z}_{\alpha}\left(\right.$ or $\left.\mathscr{Z}_{\alpha, 0}\right)$.

Corollary 14. Let $\alpha>0,0<p<\infty$. Then the pointwise multiplier $M_{u}: H^{p} \rightarrow \mathscr{Z}_{\alpha}$ is a bounded operator if and only if
(i) $u=0$ if $\alpha<2+1 / p$;
(ii) $u \in H^{\infty}$ if $\alpha=2+1 / p$;
(iii) $\sup _{z \in D}\left(1-|z|^{2}\right)^{\alpha-2-1 / p}|u(z)|<\infty$ if $\alpha>2+1 / p$. 
Corollary 15. Let $\alpha>0,0<p<\infty$. Then the pointwise multiplier $M_{u}: H^{p} \rightarrow \mathscr{Z}_{\alpha, 0}$ is a bounded operator if and only if $M_{u}: H^{p} \rightarrow \mathscr{Z}_{\alpha, 0}$ is a compact operator if and only if $M_{u}: H^{p} \rightarrow \mathscr{Z}_{\alpha}$ is a compact operator if and only if

(i) $u=0$ if $\alpha \leq 2+1 / p$,

(ii) $\lim _{|z| \rightarrow 1^{-}}\left(1-|z|^{2}\right)^{\alpha-2-1 / p}|u(z)|=0$ if $\alpha>2+1 / p$.

\section{Acknowledgments}

The research was supported by Special Fund of Colleges and Universities in Fujian Province (no. JK2012010) and Natural Science Foundation of Fujian Province, China (no. 2009J01004).

\section{References}

[1] K. Madigan and A. Matheson, "Compact composition operators on the Bloch space," Transactions of the American Mathematical Society, vol. 347, no. 7, pp. 2679-2687, 1995.

[2] K. M. Madigan, "Composition operators on analytic Lipschitz spaces," Proceedings of the American Mathematical Society, vol. 119, no. 2, pp. 465-473, 1993.

[3] W. Smith, "Composition operators between Bergman and Hardy spaces," Transactions of the American Mathematical Society, vol. 348, no. 6, pp. 2331-2348, 1996.

[4] R. Yoneda, "The composition operators on weighted Bloch space," Archiv der Mathematik, vol. 78, no. 4, pp. 310-317, 2002.

[5] R. J. Fleming and J. E. Jamison, Isometries on Banach Spaces: Function Spaces, vol. 129 of Chapman \& Hall/CRC Monographs and Surveys in Pure and Applied Mathematics, Chapman \& Hall/CRC, Boca Raton, Fla, USA, 2003.

[6] M. D. Contreras and A. G. Hernández-Díaz, "Weighted composition operators on Hardy spaces," Journal of Mathematical Analysis and Applications, vol. 263, no. 1, pp. 224-233, 2001.

[7] Z. Cučković and R. Zhao, "Weighted composition operators on the Bergman space," Journal of the London Mathematical Society, vol. 70, no. 2, pp. 499-511, 2004.

[8] J. Laitila, "Weighted composition operators on BMOA," Computational Methods and Function Theory, vol. 9, no. 1, pp. 27-46, 2009.

[9] S. Ohno and R. Zhao, "Weighted composition operators on the Bloch space," Bulletin of the Australian Mathematical Society, vol. 63, no. 2, pp. 177-185, 2001.

[10] S. Ye, "A weighted composition operator on the logarithmic Bloch space," Bulletin of the Korean Mathematical Society, vol. 47, no. 3, pp. 527-540, 2010.

[11] S. Ye and Q. Hu, "Weighted composition operators on the Zygmund space," Abstract and Applied Analysis, vol. 2012, Article ID 462482, 18 pages, 2012.

[12] Z. Čučković and R. Zhao, "Weighted composition operators between different weighted Bergman spaces and different Hardy spaces," Illinois Journal of Mathematics, vol. 51, no. 2, pp. 479-498, 2007.

[13] S. Ohno, K. Stroethoff, and R. Zhao, "Weighted composition operators between Bloch-type spaces," The Rocky Mountain Journal of Mathematics, vol. 33, no. 1, pp. 191-215, 2003.

[14] A. K. Sharma, "Products of multiplication, composition and differentiation between weighted Bergman-Nevanlinna and Bloch-type spaces," Turkish Journal of Mathematics, vol. 35, no. 2, pp. 275-291, 2011.
[15] A. K. Sharma and S.-I. Ueki, "Composition operators from Nevanlinna type spaces to Bloch type spaces," Banach Journal of Mathematical Analysis, vol. 6, no. 1, pp. 112-123, 2012.

[16] S. Stević, "Weighted composition operators from BergmanPrivalov-type spaces to weighted-type spaces on the unit ball," Applied Mathematics and Computation, vol. 217, no. 5, pp. 19391943, 2010.

[17] S. Stević and A. K. Sharma, "Essential norm of composition operators between weighted Hardy spaces," Applied Mathematics and Computation, vol. 217, no. 13, pp. 6192-6197, 2011.

[18] S. Stević and A. K. Sharma, "Composition operators from the space of Cauchy transforms to Bloch and the little Bloch-type spaces on the unit disk," Applied Mathematics and Computation, vol. 217, no. 24, pp. 10187-10194, 2011.

[19] S. Ye, "A weighted composition operator between different weighted Bloch-type spaces," Acta Mathematica Sinica. Chinese Series, vol. 50, no. 4, pp. 927-942, 2007.

[20] S. Ye, "Weighted composition operators from $F(p, q, s)$ into logarithmic Bloch space," Journal of the Korean Mathematical Society, vol. 45, no. 4, pp. 977-991, 2008.

[21] S. Ye, "Weighted composition operators between the little logarithmic Bloch space and the $\alpha$-Bloch space," Journal of Computational Analysis and Applications, vol. 11, no. 3, pp. 443450, 2009.

[22] S. Ye, "Norm of composition followed by differentiation from logarithmic Bloch space to theweighted-type space," Ars Combrination. In press.

[23] F. Colonna and S. Li, "Weighted composition operators from Hardy spaces into logarithmic Bloch spaces," Journal of Function Spaces and Applications, vol. 2012, Article ID 454820, 20 pages, 2012.

[24] P. L. Duren, Theory of $H^{p}$ Spaces, vol. 38 of Pure and Applied Mathematics, Academic Press, New York, NY, USA, 1970.

[25] J. B. Garnett, Bounded Analytic Functions, vol. 236 of Graduate Texts in Mathematics, Springer, New York, NY, USA, Revised 1st edition, 2007.

[26] A. Zygmund, Trigonometric Series, Cambridge University Press, London, UK, 1959.

[27] K. H. Zhu, "Bloch type spaces of analytic functions," The Rocky Mountain Journal of Mathematics, vol. 23, no. 3, pp. 1143-1177, 1993.

[28] B. R. Choe, H. Koo, and W. Smith, "Composition operators on small spaces," Integral Equations and Operator Theory, vol. 56, no. 3, pp. 357-380, 2006.

[29] S. Li and S. Stević, "Generalized composition operators on Zygmund spaces and Bloch type spaces," Journal of Mathematical Analysis and Applications, vol. 338, no. 2, pp. 1282-1295, 2008.

[30] S. Li and S. Stević, "Products of Volterra type operator and composition operator from $H^{\infty}$ and Bloch spaces to Zygmund spaces," Journal of Mathematical Analysis and Applications, vol. 345, no. 1, pp. 40-52, 2008.

[31] S. Li and S. Stević, "Weighted composition operators from Zygmund spaces into Bloch spaces," Applied Mathematics and Computation, vol. 206, no. 2, pp. 825-831, 2008.

[32] C. C. Cowen and B. D. MacCluer, Composition Operators on Spaces of Analytic Functions, Studies in Advanced Mathematics, CRC Press, Boca Raton, Fla, USA, 1995. 


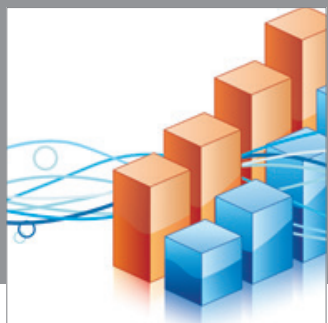

Advances in

Operations Research

mansans

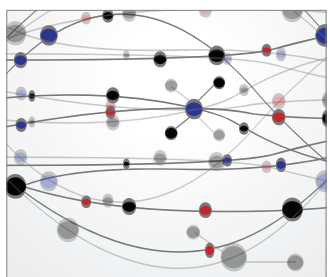

The Scientific World Journal
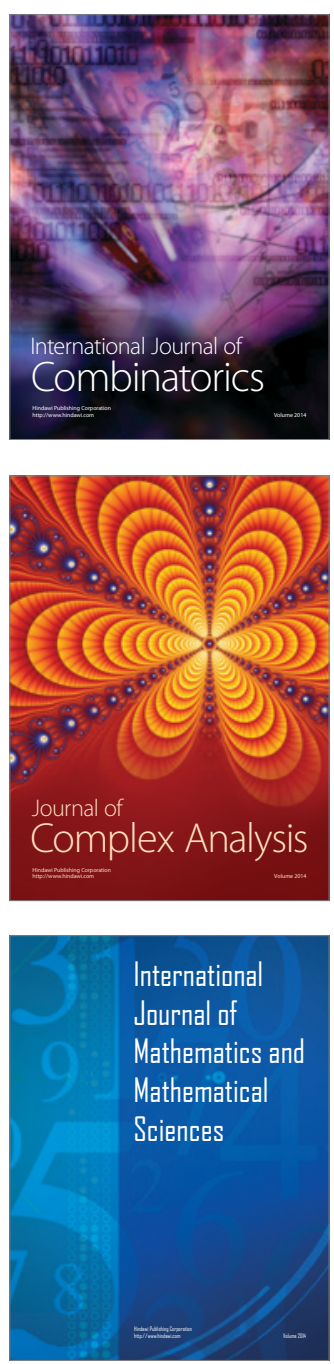
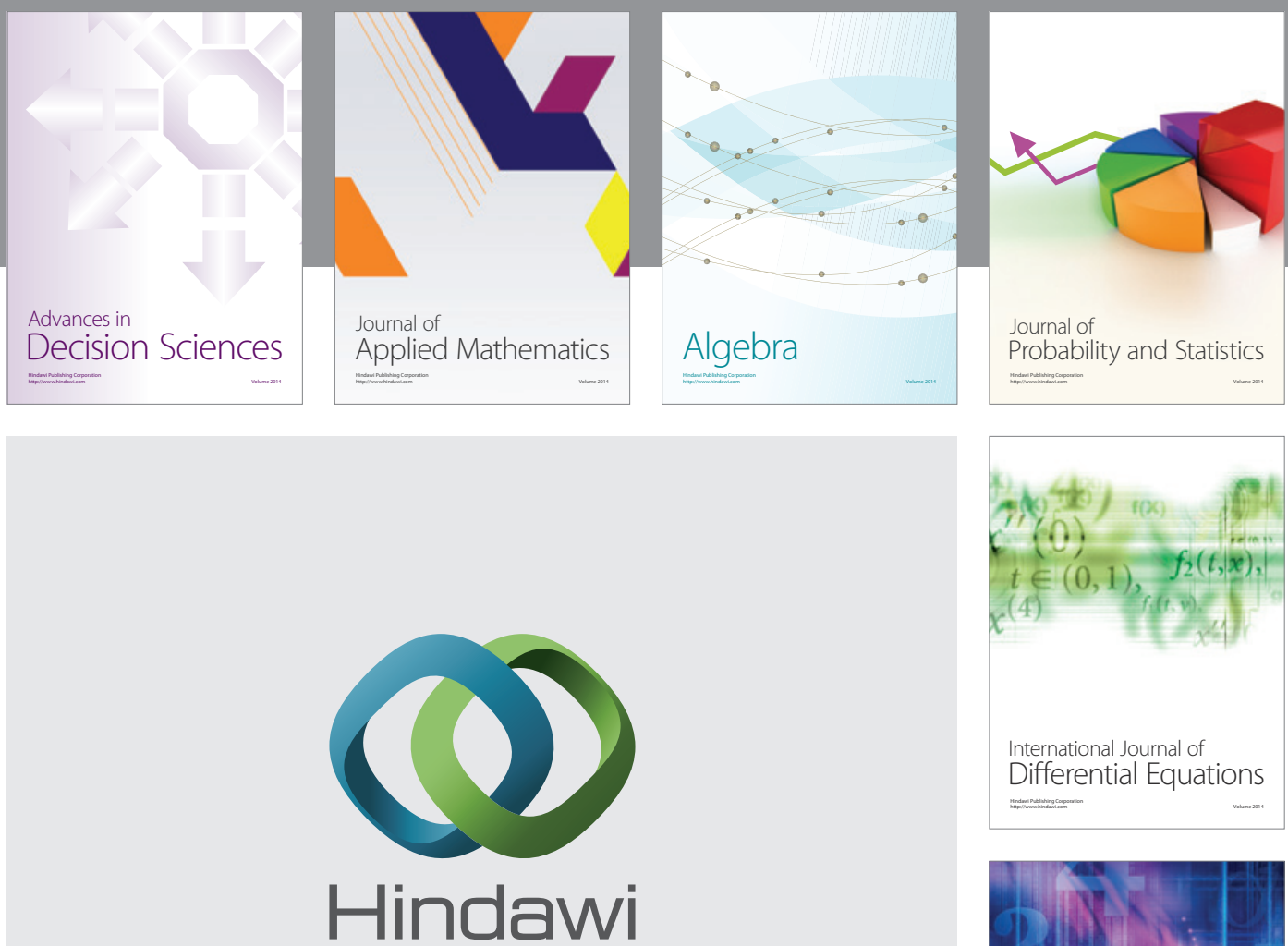

Submit your manuscripts at http://www.hindawi.com
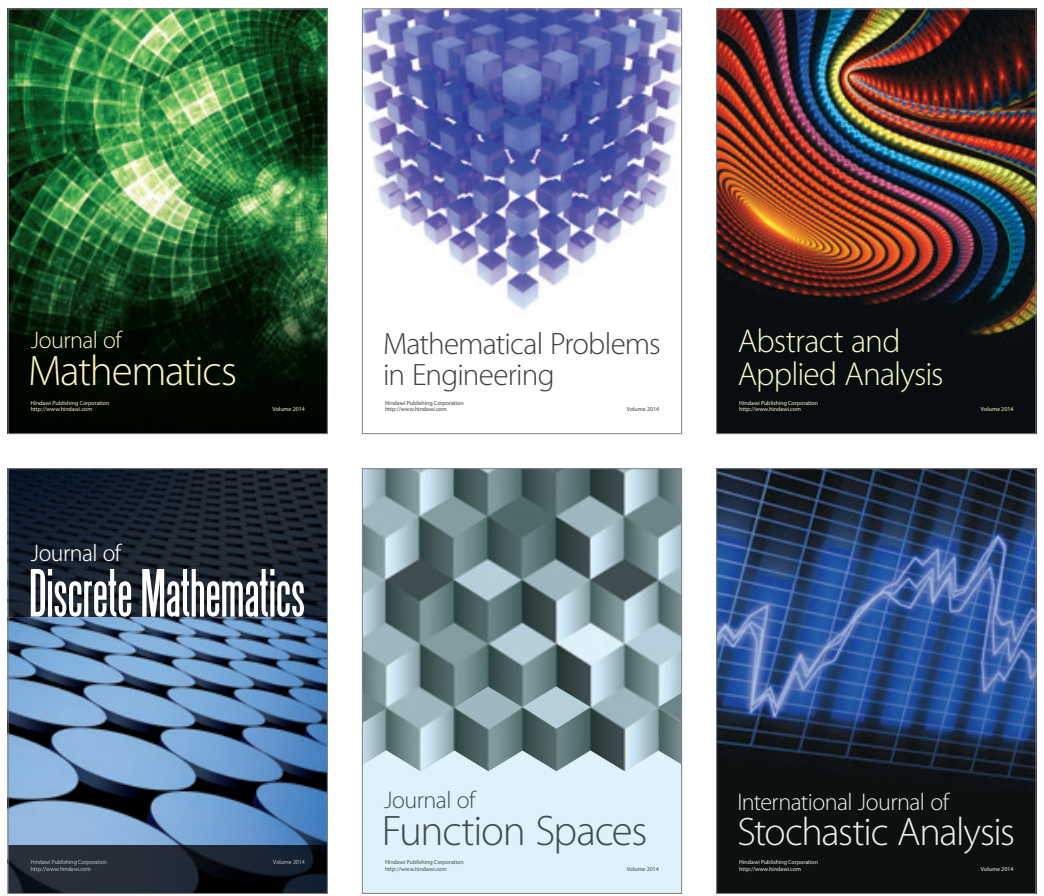

Journal of

Function Spaces

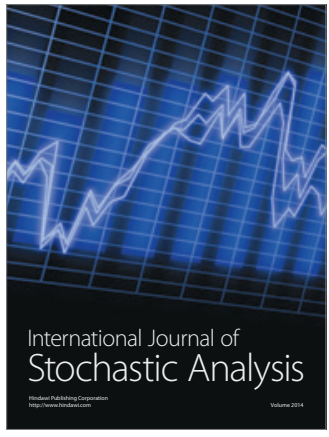

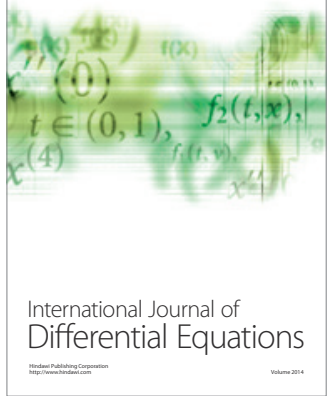
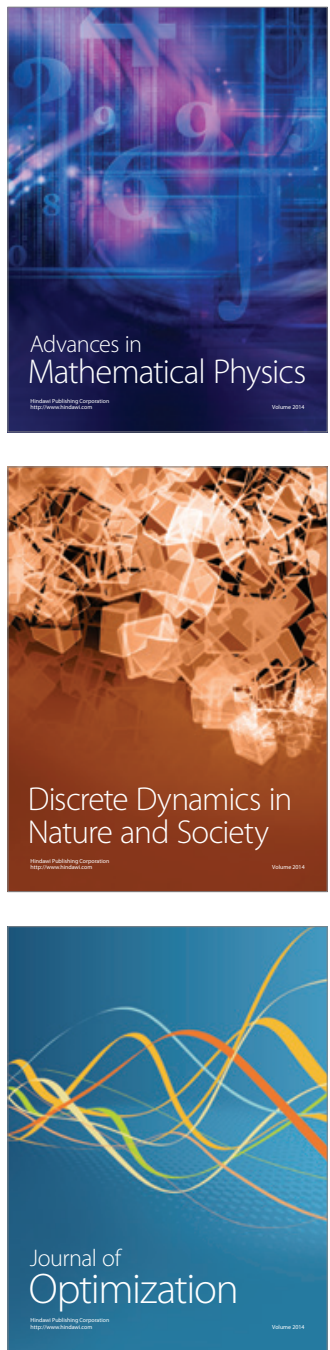